\title{
Exogenous addition of histidine reduces copper availability in the yeast Saccharomyces cerevisiae
}

\author{
Daisuke Watanabe, Rie Kikushima, Miho Aitoku, Akira Nishimura, Iwao Ohtsu, Ryo Nasuno, and Hiroshi \\ Takagi* \\ Graduate School of Biological Sciences, Nara Institute of Science and Technology, 8916-5 Takayama, Ikoma, Nara 630-0192, Japan. \\ * Corresponding Author: Hiroshi Takagi, Graduate School of Biological Sciences, Nara Institute of Science and Technology, 8916-5 \\ Takayama, Ikoma; Nara 630-0192, Japan; Tel: +81 74372 5420; Fax: +81 74372 5429; E-mail: hiro@bs.naist.jp
}

\begin{abstract}
The basic amino acid histidine inhibited yeast cell growth more severely than lysine and arginine. Overexpression of CTR1, which encodes a high-affinity copper transporter on the plasma membrane, or addition of copper to the medium alleviated this cytotoxicity. However, the intracellular level of copper ions was not decreased in the presence of excess histidine. These results indicate that histidine cytotoxicity is associated with low copper availability inside cells, not with impaired copper uptake. Furthermore, histidine did not affect cell growth under limited respiration conditions, suggesting that histidine cytotoxicity is involved in deficiency of mitochondrial copper.
\end{abstract}

\author{
doi: $10.15698 / \operatorname{mic} 2014.07 .154$ \\ Received originally: 04.04.2014; \\ in revised form: 09.06.2014, \\ Accepted 19.06.2014 \\ Published 07.07.2014. \\ Keywords: yeast Saccharomyces \\ cerevisiae, basic amino acids, \\ histidine cytotoxicity, copper \\ transporter Ctr1, mitochondrial \\ respiration.
}

\section{INTRODUCTION}

Free amino acids play pivotal roles as building blocks of proteins, as intermediates in metabolism, and also as regulators of a wide variety of cellular functions. In our previous studies, several amino acids including proline and arginine show cryoprotective activity in the budding yeast Saccharomyces cerevisiae [1-3]. We recently showed that, under oxidative stress conditions, increased conversion of proline into arginine led to the flavoprotein Tah18-dependent synthesis of nitric oxide, which confers stress tolerance on $S$. cerevisiae cells [4-6]. Although other charged amino acids, such as lysine and glutamate, also effectively enhance the freeze tolerance for yeast cells [1], the mechanism underlying the freezing stress tolerance by these amino acids remained unclear. In contrast, intracellular excessive levels of basic amino acids (lysine, arginine, and histidine) in mammals were reported to induce toxicity leading to gastrointestinal diseases, such as hepatomegaly and acute pancreatitis [7-9]. Although pleiotropic disorders in lipid metabolism, protein synthesis, and mitochondrial functions have been observed in cells damaged by basic amino acids, their primary cellular effects are not fully understood.

Basic amino acids are incorporated via three similar permeases, Can1, Lyp1, and Alp1, which share $60-65 \%$ sequence identity with each other [10]. Can1 was originally identified as an arginine permease [11], though it has been also reported to transport lysine, histidine, and ornithine with lower affinities [12]. An ortholog of Can1 in Candida albicans actively transports lysine, arginine, and histidine [13]. Lyp1 efficiently mediates only lysine transport [14, 15].
Overexpression of $A L P 1$ leads to specific uptake of arginine, although it is unclear whether this gene is expressed under physiological conditions [15]. While these permeases transport amino acids with substrate preferences, the general amino-acid permease Gap1 is a transporter for all of $20 \mathrm{~L}$-forms and also D-forms of the common $\alpha$-amino acids, as well as other related compounds, such as citrulline, ornithine, $\gamma$-aminobutyric acid (GABA), and polyamines [16-19]. Gap1 is most closely related to Hip1 in terms of amino acid sequence, although Hip1 seems to be a rather specific permease for histidine $[15,20,21]$. Recent comprehensive studies revealed that single overexpression of these permeases decreases the growth rate $[22,23]$, suggesting that $S$. cerevisiae can be utilized as a model to analyze the cytotoxicity caused by excess basic amino acids.

Among basic amino acids, histidine is especially related to copper transport [24]. Since the discovery of copper(II)bis(L-histidinato) complex in human blood [25], extensive research has been performed to determine its physiological roles. Consequently, histidine was found to facilitate copper uptake in hepatic, placental, and brain cells [26-28] by removing copper from albumin, which physically inhibits incorporation of copper ions [29]. The copper(II)-bis(Lhistidinato) complex has thus been applied for the treatment of Menkes disease and hypertrophic cardioencephalomyopathy, both of which are closely associated with copper deficiency [30, 31]. In S. cerevisiae, copper uptake is mediated by the high-affinity transporters Ctr1 and Ctr3 and a ferric/cupric reductase Fre1, which oxidizes copper(II) into usable copper(I) ions in advance of their uptake 
[32-34]. To maintain copper homeostasis, the CTR1, CTR3, and FRE1 genes are upregulated or downregulated under copper starvation or excess copper conditions, respectively, via the action of the copper-sensing transcription factor Mac1 [35, 36]. Intriguingly, the mutations in the histidine biosynthetic genes of $S$. cerevisiae increase sensitivity to the excess amounts of copper, which is suppressed by addition of histidine [37]. This finding supports the idea that histidine might directly interact with copper ions in yeast cells to alleviate the copper toxicity.

To explore novel roles of free amino acids, we analyzed here the cytotoxicity caused by exogenous addition of excess basic amino acids in S. cerevisiae.

\section{RESULTS AND DISCUSSION}

To understand the mechanism by which excess of basic amino acids mediate cytotoxicity, we examined cell growth of $S$. cerevisiae under culture conditions supplemented with an elevated concentration of lysine, arginine, or histidine. As shown in Figure 1A, $5 \mathrm{mM}$ of histidine severely impaired the growth of yeast cells, although a higher concentration of lysine or arginine $(25 \mathrm{mM})$ did not affect growth. The concentrations for three basic amino acids were selected based on the intracellular contents of these amino acids in L5487 cells (Figure 1B). When the CAN1 gene, encoding basic amino acids permease on the plasma membrane $[12,15,38]$, was overexpressed, there was little effect on growth on SCGal medium that contained no excess of basic amino acids. In contrast, the overexpression of CAN1 markedly inhibited growth under elevated levels of basic amino acids. In particular, the growth of yeast cells that overexpress CAN1 was relatively slow on the medium in the presence of $5 \mathrm{mM}$ of histidine. We confirmed that the overexpression of CAN1 increased the intracellular levels of basic amino acids in SCGal medium (approximately 1.6 - to 4.1 -fold increase) (Figure 1B). Thus, these results suggest that the excess amount of intracellular basic amino acids exerts toxic effects on yeast cells. Considering that Can1 preferentially transports lysine and arginine [15], overexpression of the high-affinity histidine transporter gene HIP1 [15, 21] might enhance histidine uptake and effectively confer more severe toxicity to yeast cells in the presence of excess histidine. As histidine conferred more sensitivity to yeast cells than lysine and arginine despite having the lowest intracellular level, we further analyzed histidine cytotoxicity.

To identify multicopy suppressor genes that alleviate histidine toxicity, a yeast genomic library YEp51B [39] was introduced into $L 5487$ cells overexpressing CAN1, and the transformants were screened for growth on SCGal medium containing $10 \mathrm{mM}$ histidine. Sixteen independent genomic DNA fragments were isolated from the transformant colonies, and 25 full-length open reading frames were included in these fragments. After subcloning into pYES2, each gene was tested for its effect on the growth of L5487 cells in the presence of excess histidine. Consequently, the CTR1 gene, which encodes a high-affinity copper transporter that predominantly mediates copper uptake under low copper conditions [32], exhibited the most significant suppression of the histidine-caused growth defect (Figure 1C). Although the overexpression of CTR1 reduced cell growth in the presence of excess lysine by unknown mechanism(s), it is suggested that Ctr1 functions in alleviating histidine toxicity. Another high-affinity copper transporter gene, CTR3 [33], and a cupric reductase gene, FRE1, the latter of which is required for conversion of copper(II) to copper(I) ions prior to uptake [34], also suppressed the growth defect under histidine-excess conditions when overexpressed. In addition, deletion of the CTR1 or FRE1 gene slightly increased sensitivity to excess histidine (data not shown). These results indicate that the histidine cytotoxicity in $S$. cerevisiae is alleviated by enhancement of copper uptake. In agreement with these results, the growth defect caused by $5 \mathrm{mM}$ histidine became significantly more severe when the concentration of $\mathrm{CuSO}_{4}$ in SD medium was decreased (2.5 and $25 \mathrm{nM}$ ), although higher concentrations of $\mathrm{CuSO}_{4}$ ( 2.5 and $25 \mu \mathrm{M}$ ) suppressed the histidine toxicity (Figure 1D). It has been well studied that histidine directly binds to copper(II) to form copper(II)-bis(L-histidinato) complex under physiological conditions as in human blood [24]. However, considering that the overexpression of CAN1 enhanced histidine toxicity (Figure $1 \mathrm{~A}$ ), it seems unlikely that the elevated level of histidine simply chelates copper(II) ions outside of the cells to inhibit copper uptake. Instead, excess histidine might interact with copper(I) ions to reduce the availability of copper after incorporation into yeast cells. To verify this hypothesis, we quantified the intracellular level of copper ions. Although cell growth was delayed starting after 4-hour incubation with 5 or $10 \mathrm{mM}$ histidine (data not shown), intracellular copper ions were slightly increased in the histidine-treated cells (Figure 1E). Therefore, our data consistently demonstrate that an excess level of histidine enhances copper uptake but impairs copper availability in yeast cells. Regarding the CTR1overexpressing cells (Figure 1C), sufficient copper ions might be incorporated into cells in bioavailable forms and thus contributed to relieving histidine toxicity. In a similar manner as clioquinol [40], histidine may act as both a chelator and an ionophore: histidine chelates copper(II) outside of the cell and is taken in as a complex, which may facilitate uptake of copper ions, though copper ions in this complex are not bioavailable. Additionally, it is possible that histidine-induced copper deficiency upregulates expression of copper transporters, which may increase copper uptake.

What functions of copper does excess histidine inhibit? Intracellular copper is distributed to distinct target proteins via specific cytosolic copper chaperons, such as Atx1, Ccs1, and Cox17. Atx1 assists in the transport of copper to the cell-surface iron uptake protein Fet3 through the function of Ccc2, which has copper-transporting ATPase activity, on the post-Golgi vesicle [41]. Ccs1 delivers copper specifically to the superoxide dismutase Sod1, which scavenges reactive oxygen species in the intermembrane space of mitochondria $[42,43]$. Another copper chaperon Cox17 transfers copper to the mitochondrial inner membrane proteins 
A

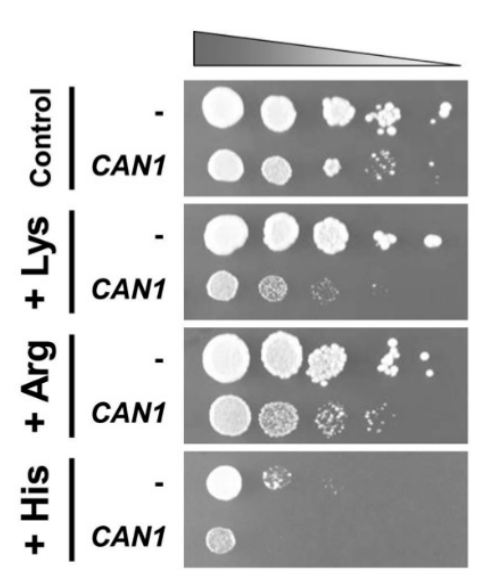

C

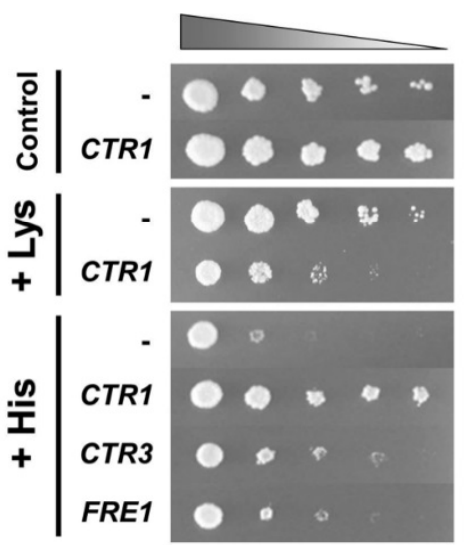

E

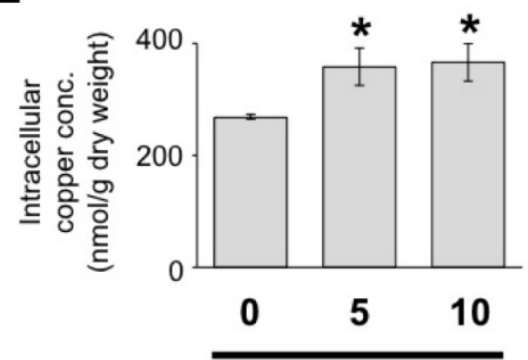

His (mM)
B

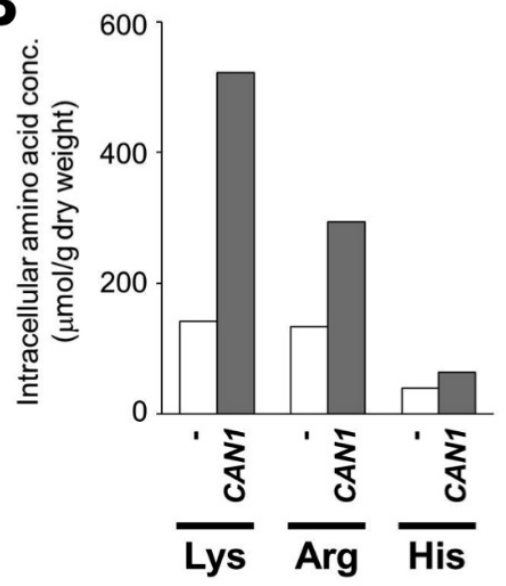

D

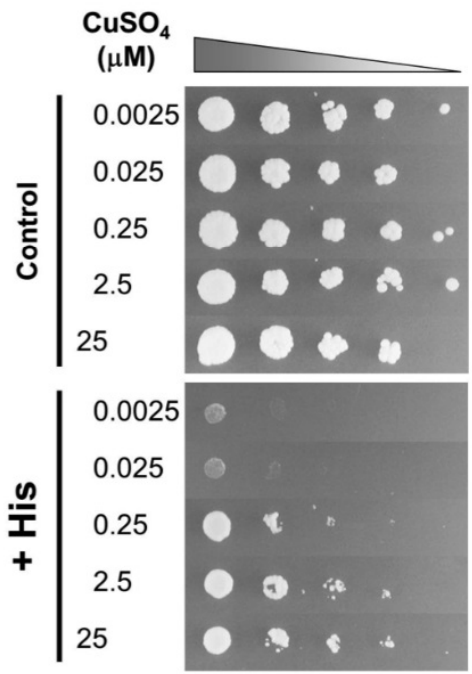

$\mathbf{F}$

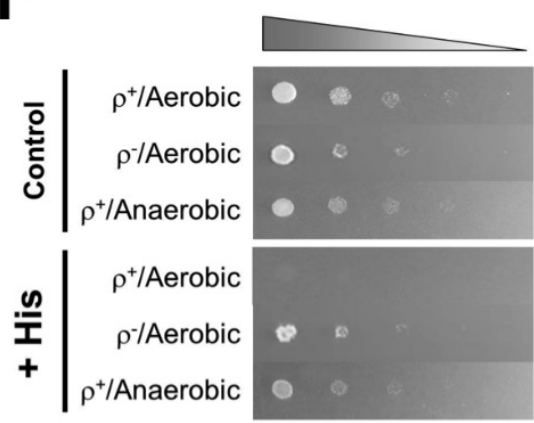

FIGURE 1: The effect of excess histidine on yeast cell growth and copper uptake. (A) Growth phenotypes of $S$. cerevisiae ¿1278b wild-type strain L5487 (complemented with pRS415 (LEU2)) carrying an empty vector PYES2 (URA3) or PYES2-CAN1 (URA3). After overnight cultivation in SC-Leu-Ura liquid medium, approximately $10^{6}$ cells of each strain, and serial dilutions of $10^{-1}$ to $10^{-4}$ (from left to right) were spotted and incubated onto SCGal-Leu-Ura-Lys-Arg-His agar medium in the absence (Control) and presence of excess basic amino acids ( $25 \mathrm{mM}$ lysine (+ Lys), $25 \mathrm{mM}$ arginine (+ Arg), or $5 \mathrm{mM}$ histidine (+ His)). (B) Intracellular contents of basic amino acids in strain L5487 (complemented with pRS415) carrying an empty vector pYES2 (white) or pYES2-CAN1 (gray). The values are the means of two independent experiments that produced similar results. (C) Growth phenotypes of strain L5487 (complemented with pRS415) carrying an empty vector pYES2, pYES2-CTR1, pYES2CTR3, and pYES2-FRE1. After overnight cultivation in SC-LeuUra liquid medium, approximately $10^{6}$ cells of each strain, and serial dilutions of $10^{-1}$ to $10^{-4}$ (from left to right) were spotted and incubated onto SCGal-Leu-Ura-Lys-Arg-His agar medium in the absence (Control) and presence of excess levels of basic amino acids (25 $\mathrm{mM}$ lysine (+ Lys) or $5 \mathrm{mM}$ histidine (+ His)). (D) Growth phenotypes of strain L5487 (complemented with pRS415 and pRS416 (URA3)) under various concentrations of copper. After overnight cultivation in SD liquid medium, which contains 0.25 $\mu \mathrm{M} \mathrm{CuSO}{ }_{4}$, approximately $10^{6}$ cells of each strain, and serial dilutions of $10^{-1}$ to $10^{-4}$ (from left to right) were spotted and incubated onto SD-Cu agar medium in the presence of $0.0025,0.025,0.25,2.5$, or $25 \mu \mathrm{M} \mathrm{CuSO}_{4}$ and in the absence (Control) and presence of $5 \mathrm{mM}$ histidine (+ His). (E) Intracellular levels of copper ions of strain L5487 (complemented with pRS415 and pRS416) in the absence ( $0 \mathrm{mM} \mathrm{His}$ ) and presence of excess histidine (5 or $10 \mathrm{mM}$ His). The values are the means and standard deviations of three independent experiments. Asterisks indicate a significant increase in copper levels compared to the control sample $(0 \mathrm{mM}$ His) $(p<0.05)$. (F) Growth phenotypes of strain L5487 (complemented with pRS415 and pRS416) (shown as $\rho^{+}$) and its spontaneous $\rho^{-}$mutant. After overnight cultivation in SD liquid medium, approximately $10^{6}$ cells of each strain, and serial dilutions of $10^{-1}$ to $10^{-4}$ (from left to right) were spotted and incubated onto SD agar medium in the absence (Control) and presence of $5 \mathrm{mM}$ histidine (+ His) under aerobic or anaerobic conditions. 
Cox11 and Sco1, both of which are essential for assembly of cytochrome $c$ oxidase, which is the last enzyme in the respiratory electron transport chain [44]. In this study, we tested whether histidine cytotoxicity might be mediated by defective mitochondrial functions due to reduced copper availability. As shown in Figure 1F, L5487 cells were clearly sensitive to $5 \mathrm{mM}$ histidine on SD plates, although histidine cytotoxicity was completely abrogated by a spontaneous cytoplasmic petite $\left(\rho^{-}\right)$mutation, which inactivates mitochondrial respiratory functions. It is also worth noting that yeast cells showed similar growth phenotypes in the absence or presence of excess histidine under anaerobic conditions (Figure 1F). Thus, histidine cytotoxicity was observed only when mitochondrial aerobic respiration should be functional. We hypothesize that the deficiency of the Cox17-bound copper ions due to excess of intracellular histidine might cause the abnormal assembly of cytochrome $c$ oxidase complex in aerobically growing cells, leading to the observed toxic effect. This might be supported by the fact that the disturbance of cytochrome $c$ oxidase induces apoptosis-like cell death in S. cerevisiae [45]. However, we cannot rule out the possibility that defective mitochondrial respiration reduces incorporation of histidine by some unknown mechanism(s), and hence, the excess level of histidine did not elicit the toxicity in $\rho^{-}$cells under aerobic conditions or in $\rho^{+}$cells under anaerobic conditions.

In this study, we discovered the cytotoxicity of excess histidine in S. cerevisiae, which is tightly associated with the reduced availability of intracellular copper ions. Similarly, Pearce and Sherman [37] revealed that intracellular histidine synthesis is required for the detoxification of excess copper. Both studies commonly suggest that intracellular histidine has a novel and important role in copper homeostasis.

\section{MATERIALS AND METHODS \\ Strains and Plasmids}

The $S$. cerevisiae strain used in this study was $\Sigma 1278 \mathrm{~b}$ wildtype strain L5487 (MATa ura3-52 leu2::hisG), which was generously provided by Gerald Fink (Whitehead Institute). A spontaneous cytoplasmic petite $\left(\rho^{-}\right)$mutant of L5487 was isolated, according to Fox et al. [46]. Escherichia coli strain DH5 $\alpha$ (F- $\lambda$ Ф80lacZDM15 $\triangle$ (lacZYA argF)U169 deoR recA1 endA1 hsdR17 $\left(r_{k}^{-} m_{k}{ }^{+}\right)$supE44 thi-1 gyrA96) was used to subclone the yeast gene and construct plasmids. Low-copy plasmids pRS415 and pRS416 [24] were used to complement auxotrophic mutations ura3 and leu2, respectively. A galactose-inducible plasmid pYES2 (Life Technologies) was used for overexpression of the CAN1, CTR1, CTR3, and FRE1 gene. A $2 \mu \mathrm{m}$-based yeast genomic library YEp51B [12] was used to identify multicopy suppressor genes of CAN1-overexpressing L5487 strain.

\section{Culture Media}

The media used for growth of $S$. cerevisiae were a synthetic complete medium, SC ( $2 \%$ glucose, $0.67 \%$ yeast nitrogen base without amino acids (Difco), supplemented with synthetic drop-out amino acid and nucleotide mixture as required), and a synthetic defined medium, SD ( $2 \%$ glucose, $0.67 \%$ yeast $\mathrm{ni}$ trogen base without amino acids (Difco)). For overexpression of CAN1, CTR1, CTR3, and FRE1, SC with galactose as a carbon source (SCGal) ( $2 \%$ galactose, $0.67 \%$ yeast nitrogen base without amino acids (Difco), supplemented with synthetic dropout amino acid and nucleotide mixture as required) was used. To evaluate the effect of copper sulfate $\left(\mathrm{CuSO}_{4}\right)$ addition, SD$\mathrm{Cu}$ media ( $2 \%$ glucose, $0.67 \%$ yeast nitrogen base without amino acids and copper (ForMedium)) containing different concentrations of $\mathrm{CuSO}_{4}$ were used. All experiments were performed at $30^{\circ} \mathrm{C}$, and all growth media were adjusted to $\mathrm{pH}$ 6.5 with HEPES buffer $(\mathrm{pH} \mathrm{7.0)}$ and sodium hydroxide. When necessary, $2 \%$ agar was added to solidify the medium. For anaerobic cultivation, the inoculated plates were incubated with $\mathrm{O}_{2}$-absorber/ $/ \mathrm{CO}_{2}$-generator AnaeroPouch-Anaero (Mitsubishi Gas Chemical Company) and $\mathrm{O}_{2}$ indicators. The $E$. coli recombinant strains were grown in Luria-Bertani complete medium containing $50 \mu \mathrm{g} / \mathrm{ml}$ ampicillin or $\mathrm{M9}$ minimal medium plus $2 \%$ Casamino acids containing $50 \mu \mathrm{g} / \mathrm{ml}$ ampicillin. If necessary, $2 \%$ agar was added to solidify the medium.

\section{Measurements of Intracellular Amino Acids Levels}

According to a method described previously [3], intracellular amino acids were extracted by boiling from log-phase cells cultivated in SCGal-Leu-Ura liquid medium, and were subsequently quantified with an amino acid analyzer AminoTac JLC500/V (JEOL).

\section{Determination of Intracellular Copper lons}

Cell lysates were prepared from log-phase cells cultivated in SC-Leu-Ura-Lys-Arg-His liquid medium, and copper ions concentrations were determined by the CUPRAC-BCS assay [25].

\section{ACKNOWLEDGMENTS}

We are grateful to Dr. Gerald R. Fink and the NBRP of the MEXT, Japan, for providing the $S$. cerevisiae $\Sigma 1278 \mathrm{~b}$ wild-type strain L5487 and a high-copy yeast genomic DNA library YEp51B, respectively.

\section{CONFLICT OF INTEREST}

The authors declare no conflict of interest.

\section{COPYRIGHT}

(C) 2014 Watanabe et al. This is an open-access article released under the terms of the Creative Commons Attribution (CC BY) license, which allows the unrestricted use, distribution, and reproduction in any medium, provided the original author and source are acknowledged.

Please cite this article as: Daisuke Watanabe, Rie Kikushima, Miho Aitoku, Akira Nishimura, Iwao Ohtsu, Ryo Nasuno, and Hiroshi Takagi (2014). Exogenous addition of histidine reduces copper availability in the yeast Saccharomyces cerevisiae. Microbial Cell 1(7): 241-246. doi: 10.15698/mic2014.07.154 


\section{REFERENCES}

1. Takagi $\mathrm{H}$, Iwamoto $\mathrm{F}$, and Nakamori S (1997). Isolation of freezetolerant laboratory strains of Saccharomyces cerevisiae from prolineanalogue-resistant mutants. Appl Microbiol Biotechnol 47(4): 405411

2. Morita $Y$, Nakamori $S$, and Takagi $H$ (2002). Effect of proline and arginine metabolism on freezing stress of Saccharomyces cerevisiae. $\mathbf{J}$ Biosci Bioeng 94(5): 390-394.

3. Morita Y, Nakamori S, and Takagi H (2003). L-Proline accumulation and freeze tolerance in Saccharomyces cerevisiae are caused by a mutation in the PRO1 gene encoding ?-glutamyl kinase. Appl Environ Microbiol 69(1): 212-219.

4. Nishimura A, Kotani T, Sasano $Y$, and Takagi H (2010). An antioxidative mechanism mediated by the yeast $\mathrm{N}$-acetyltransferase Mpr1: oxidative stress-induced arginine synthesis and its physiological role. FEMS Yeast Res 10(6): 687-698.

5. Sasano Y, Haitani Y, Hashida K, Ohtsu I, Shima J, and Takagi H (2012). Enhancement of the proline and nitric oxide synthetic pathway improves fermentation ability under multiple baking-associated stress conditions in industrial baker's yeast. Microb Cell Fact 11: 40.

6. Nishimura A, Kawahara N, and Takagi H (2013). The flavoprotein Tah18-dependent NO synthesis confers high-temperature stress tolerance on yeast cells. Biochem Biophys Res Commun 430(1): 137-143.

7. Solomon JK and Geison RL (1978). Effect of excess dietary Lhistidine on plasma cholesterol levels in weanling rats. J Nutr 108(6): 936-943.

8. Mizunuma T, Kawamura S, and Kishino $Y$ (1984). Effects of injecting excess arginine on rat pancreas. J Nutr 114(3): 467-471.

9. Biczó G, Hegyi P, Dósa S, Shalbuyeva N, Berczi S, Sinervirta R, Hracskó Z, Siska A, Kukor Z, Jármay K, Venglovecz V, Varga IS, Iványi B, Alhonen L, Wittmann T, Gukovskaya A, Takács T, and Rakonczay Z Jr (2011). The crucial role of early mitochondrial injury in L-lysineinduced acute pancreatitis. Antioxid Redox Signal 15(10): 2669-2681.

10. Schmidt KH, Viebranz E, Doerfler L, Lester C, and Rubenstein A (2010). Formation of complex and unstable chromosomal translocations in yeast. PLoS One 5(8): e12007.

11. Grenson M, Mousset M, Wiame JM, and Bechet J (1966). Multiplicity of the amino acid permeases in Saccharomyces cerevisiae. I. Evidence for a specific arginine-transporting system. Biochim Biophys Acta 127(2): 325-338.

12. Cooper TG (1982). Nitrogen metabolism and Saccharomyces cerevisiae. In: Strathern JN, Jones EW, and Broach JR, editors. The molecular biology of the yeast Saccharomyces: metabolism and gene expression. Cold Spring Harbor Laboratory, New York; pp 339-461.

13. Sychrová H and Souciet JL (1994). CAN1, a gene encoding a permease for basic amino acids in Candida albicans. Yeast 10(12): 16471651.

14. Sychrova $\mathrm{H}$ and Chevallier MR (1993). Cloning and sequencing of the Saccharomyces cerevisiae gene LYP1 coding for a lysine-specific permease. Yeast 9(7): 771-782.

15. Regenberg B, Düring-Olsen L, Kielland-Brandt MC, and Holmberg S (1999). Substrate specificity and gene expression of the amino-acid permeases in Saccharomyces cerevisiae. Curr Genet 36(6): 317-328.

16. Grenson $M$ and Acheroy $B$ (1982). Mutations affecting the activity and the regulation of the general amino-acid permease of Saccharomyces cerevisiae. Localisation of the cis-acting dominant pgr regulatory mutation in the structural gene of this permease. Mol Gen Genet 188(2): 261-265.
17. Jauniaux JC and Grenson M. (1990). GAP1, the general amino acid permease gene of Saccharomyces cerevisiae. Nucleotide sequence, protein similarity with the other bakers yeast amino acid permeases, and nitrogen catabolite repression. Eur J Biochem 190(1): 39-44.

18. Correa García S, Bermúdez Moretti M, Ramos E, and Batlle A. (1997). Carbon and nitrogen sources regulate delta-aminolevulinic acid and ?-aminobutyric acid transport in Saccharomyces cerevisiae. Int J Biochem Cell Biol 29(8-9): 1097-1101.

19. Uemura T, Kashiwagi K, and Igarashi K (2005). Uptake of putrescine and spermidine by Gap1p on the plasma membrane in Saccharomyces cerevisiae. Biochem Biophys Res Commun 328(4): 10281033.

20. Crabeel M and Grenson M (1970). Regulation of histidine uptake by specific feedback inhibition of two histidine permeases in Saccharomyces cerevisiae. Eur J Biochem 14(1): 197-204.

21. Tanaka J and Fink GR (1985). The histidine permease gene (HIP1) of Saccharomyces cerevisiae. Gene 38(1-3): 205-214.

22. Sopko R, Huang D, Preston N, Chua G, Papp B, Kafadar K, Snyder M, Oliver SG, Cyert M, Hughes TR, and Andrews B (2006). Mapping pathways and phenotypes by systematic gene overexpression. Mol Cell 21(3): 319-30.

23. Yoshikawa K, Tanaka T, Ida Y, Furusawa C, Hirasawa T, and Shimizu $\mathrm{H}$ (2011). Comprehensive phenotypic analysis of single-gene deletion and overexpression strains of Saccharomyces cerevisiae. Yeast 28(5): 349-361.

24. Deschamps P, Kulkarnip P, Gautam-Basak M, and Sarkar B (2005). The saga of copper(II)-L-histidine. Coord Chem Rev 249(9-10): 895909.

25. Sarkar B and Kruck TPA (1966). Copper-amino acid complexes in human serum. In: Peisach J, Aisen P, and Blumberg WE, editors. Biochemistry of copper. Academic Press, New York; pp 183-196.

26. Darwish HM, Cheney JC, Schmitt RC, and Ettinger MJ (1984). Mobilization of copper(II) from plasma components and mechanisms of hepatic copper transport. Am J Physiol 246(1 Pt 1): G72-G79.

27. Hartter DE and Barnea A (1988). Brain tissue accumulates ${ }^{67}$ copper by two ligand-dependent saturable processes. A high affinity, low capacity and a low affinity, high capacity process. J Biol Chem 263(2): 799-805.

28. Mas A and Sarkar B (1992). Uptake of ${ }^{67} \mathrm{Cu}$ by isolated human trophoblast cells. Biochim Biophys Acta 1135(2): 123-128.

29. McArdle HJ, Guthrie JR, Ackland ML, and Danks DM (1987). Albumin has no role in the uptake of copper by human fibroblasts. J Inorg Biochem 31(2): 123-131.

30. Sarkar B (1999). Treatment of Wilson and Menkes diseases. Chem $\operatorname{Rev}$ 99(9): 2535-2544.

31. Papadopoulou LC, Sue CM, Davidson MM, Tanji K, Nishino I, Sadlock JE, Krishna S, Walker W, Selby J, Glerum DM, Coster RV, Lyon G, Scalais E, Lebel R, Kaplan P, Shanske S, De Vivo DC, Bonilla E, Hirano $M$, DiMauro S, and Schon EA (1999). Fatal infantile cardioencephalomyopathy with COX deficiency and mutations in SCO2, a COX assembly gene. Nat Genet 23(3): 333-337.

32. Dancis A, Yuan DS, Haile D, Askwith C, Eide D, Moehle C, Kaplan J, and Klausner RD (1994). Molecular characterization of a copper transport protein in S. cerevisiae: an unexpected role for copper in iron transport. Cell 76(2): 393-402.

33. Knight SA, Labbé S, Kwon LF, Kosman DJ, and Thiele DJ (1996). A widespread transposable element masks expression of a yeast copper transport gene. Genes Dev 10(15): 1917-1929. 
34. Hassett R and Kosman DJ (1995). Evidence for $\mathrm{Cu}(\mathrm{II})$ reduction as a component of copper uptake by Saccharomyces cerevisiae. J Biol Chem 270(1): 128-134.

35. Labbé S, Zhu Z, and Thiele DJ (1997). Copper-specific transcriptional repression of yeast genes encoding critical components in the copper transport pathway. J Biol Chem 272(25): 15951-15958.

36. Yamaguchi-Iwai Y, Serpe M, Haile D, Yang W, Kosman DJ, Klausner $\mathrm{RD}$, and Dancis A (1997). Homeostatic regulation of copper uptake in yeast via direct binding of MAC1 protein to upstream regulatory sequences of FRE1 and CTR1. J Biol Chem 272(28): 17711-17718.

37. Pearce DA and Sherman F (1999). Toxicity of copper, cobalt, and nickel salts is dependent on histidine metabolism in the yeast Saccharomyces cerevisiae. J Bacteriol 181(16): 4774-4779.

38. Malínská K, Malínský J, Opekarová M, and Tanner W (2003). Visualization of protein compartmentation within the plasma membrane of living yeast cells. Mol Biol Cell 14(11): 4427-4436.

39. Akada R, Yamamoto J, and Yamashita I (1997). Screening and identification of yeast sequences that cause growth inhibition when overexpressed. Mol Gen Genet 254(3): 267-274.

40. Li C, Wang J, and Zhou B (2010). The metal chelating and chaperoning effects of clioquinol: insights from yeast studies. J Alzheimers Dis 21(4): 1249-1262.
41. Lin SJ, Pufahl RA, Dancis A, O'Halloran TV, and Culotta VC (1997). A role for the Saccharomyces cerevisiae ATX1 gene in copper trafficking and iron transport. J Biol Chem 272(14): 9215-9220.

42. Culotta VC, Klomp LW, Strain J, Casareno RL, Krems B, and Gitlin JD (1997). The copper chaperone for superoxide dismutase. J Biol Chem 272(38): 23469-23472.

43. Sturtz LA, Diekert K, Jensen LT, Lill R, and Culotta VC (2001). A fraction of yeast $\mathrm{Cu}, \mathrm{Zn}$-superoxide dismutase and its metallochaperone, CCS, localize to the intermembrane space of mitochondria. A physiological role for SOD1 in guarding against mitochondrial oxidative damage. J Biol Chem 276(41): 38084-38089.

44. Horng YC, Cobine PA, Maxfield AB, Carr HS, and Winge DR (2004). Specific copper transfer from the Cox17 metallochaperone to both Sco1 and Cox11 in the assembly of yeast cytochrome $c$ oxidase. J Biol Chem 279(34): 35334-35340.

45. Zuo X, Djordjevic JT, Bijosono Oei J, Desmarini D, Schibeci SD, Jolliffe KA, and Sorrell TC (2011). Miltefosine induces apoptosis-like cell death in yeast via Cox9p in cytochrome $c$ oxidase. Mol Pharmacol 80(3): 476-485.

46. Fox TD, Folley LS, Mulero JJ, McMullin TW, Thorsness PE, Hedin LO, and Costanzo MC (1991). Analysis and manipulation of yeast mitochondrial genes. Methods Enzymol 194: 149-165. 